\title{
New Results on Robust Model Predictive Control for Time-Delay Systems with Input Constraints
}

\author{
Qing Lu, ${ }^{1}$ Yiyong Sun, ${ }^{2}$ Qi Zhou, ${ }^{3}$ and Zhiguang Feng ${ }^{3}$ \\ ${ }^{1}$ College of Engineering, Bohai University, Jinzhou, Liaoning 121013, China \\ ${ }^{2}$ Research Institute of Intelligent Control and Systems, Harbin Institute of Technology, Harbin, Heilongjiang 150080, China \\ ${ }^{3}$ College of Information Science and Technology, Bohai University, Jinzhou, Liaoning 121013, China \\ Correspondence should be addressed to Qi Zhou; zhouqi2009@gmail.com
}

Received 20 July 2014; Accepted 8 October 2014; Published 27 November 2014

Academic Editor: Changzhu Zhang

Copyright (c) 2014 Qing Lu et al. This is an open access article distributed under the Creative Commons Attribution License, which permits unrestricted use, distribution, and reproduction in any medium, provided the original work is properly cited.

\begin{abstract}
This paper investigates the problem of model predictive control for a class of nonlinear systems subject to state delays and input constraints. The time-varying delay is considered with both upper and lower bounds. A new model is proposed to approximate the delay. And the uncertainty is polytopic type. For the state-feedback MPC design objective, we formulate an optimization problem. Under model transformation, a new model predictive controller is designed such that the robust asymptotical stability of the closedloop system can be guaranteed. Finally, the applicability of the presented results are demonstrated by a practical example.
\end{abstract}

\section{Introduction}

The ideas of model predictive control and receding horizon control have been developed since 1960s. It has been shown from [1] that model predictive control (MPC) is an effective way to handle multivariable constrained control problems, which appear in the chemical process control, the petrochemical industries, gas pipeline, and so on. In [2,3], the authors gave us an overview of the origins of model predictive control and the recent results. The original MPC technique is aimed at solving an open-loop optimization problem with constraints at every sampling instant, implementing only the first control step of solutions.

In practical control systems, parameter uncertainties cannot be avoided. In the literatures, two kinds of parameter uncertainties are often included in the uncertain systems. They are norm-bounded parameter uncertainty and polytopic parameter uncertainty. In addition, time-delay often appears in industrial processes, which results in degradation and instability in such systems [4]. References $[5,6]$ studied the networked control with time-delay; [7] investigated linear switched systems with time-varying delay. The authors in [8] discussed the problem of dissipativity analysis of stochastic neural networks systems of discrete-time form with time-varying and finite-distributed delays. The authors in [9] designed a novel output-feedback controller for the suspension systems with input delay. Moreover, there exist some physical limits, for instance power limitations and value saturation, in many industrial processes, which result in constraints on input and output. Therefore, considerable researchers have been attracted to study the robust control problem of constrained uncertain systems with state delays $[10,11]$.

Many results about MPC technique for time-delay systems have been addressed. To mention a few, in [12], the authors proposed that the control strategy for uncertain systems could be developed into a delay system via the MPC. However, it is proper only if the delay indices are known. Recently, the authors in [10] presented an improved delaydependent robust MPC to reduce the conservatism, still with a known delay. The work in [4] put forward an MPC method for time-varying state-delay systems with uncertainty and constrained control input. However, since the stability is guaranteed under the fixed constant weighting matrix at all time, the method is very limited and the conservatism may be generated.

Motivated by the above observation, the problem of MPC for time-varying delay systems with parameter uncertainties 
and input constraints is studied in this paper. We summarize the main contributions of this paper as follows. (1) The uncertainty is supposed to be polytopic uncertainty type, and the state with unknown delay with both specified upper and lower bounds is handled by an approximated model. (2) In the controller design process, for the state-feedback MPC design objective, we formulate an optimization problem over an infinite time horizon. A new model predictive controller is designed under the model transformation by approximating the state delay, such that the robust asymptotical stability of the closed-loop system is guaranteed. The existence of the controller can be expressed by the convex optimization algorithm. (3) It is shown that the approach proposed in this paper is effective and performs better with the faster response, smaller overshoot, stronger robustness and so on by a practical example.

The rest of this paper is organized as follows. Section 2 formulates the problem to be solved. Section 3 proposes an MPC method for delay systems with uncertainties and constraints. Section 4 illustrates the effectiveness of the method proposed in this paper with a practical example. This paper is concluded in Section 5.

Notation. $R^{n}$ stands for the $n$-dimensional Euclidean space, $R^{n \times m}$ denotes the set of $n \times m$ real matrices, $I_{n}$ denotes the $n \times n$ identity matrix, and $\operatorname{diag}\{\cdots\}$ denotes a block-diagonal matrix. $X>0(X \geq 0)$ denotes that the matrix $X$ is a positive definite (resp. a positive semi-definite) matrix. $X^{T}$ denotes transposition of $X$, and $X^{-1}$ denotes the inverse matrix of $X$. $H^{T}=H$, when $H$ is symmetric matrix. $\|\cdot\|$ denotes 2-norm, and $\|x\|_{P}^{2}=x^{T} P x$, where $P>0$. The symbol $*$ induces a symmetric structure in a matrix.

\section{Problem Formulation}

Consider the following system:

$$
\begin{aligned}
x(k+1) & =A(k) x(k)+\bar{A}(k) x(k-d(k))+B(k) u(k), \\
x(k) & =\phi(k), \quad k \in\left[-d_{M}, 0\right],
\end{aligned}
$$

where $x(k) \in R^{n}$ denotes the state variable with the initial condition $\phi(k) \in R^{n}, d(k)$ means the unknown value of delay units, being supposed $0 \leq d_{m} \leq d(k) \leq d_{M}$ with known integers $d_{m}$ and $d_{M}$, and $u(k) \in R^{n_{u}}$ stands for the control input variable and satisfies

$$
-\bar{u} \leq u(k) \leq \bar{u}, \quad \bar{u} \geqslant 0, \quad \forall k \in[0, \infty) .
$$

$[A(k) \bar{A}(k) B(k)]$ is unknown but belongs to a polytope $\Omega$ at each time $k$, that is

$$
\begin{aligned}
& {\left[\begin{array}{ll}
A(k) & \bar{A}(k) \\
\end{array}\right.} \\
& \quad \triangleq \operatorname{Co}\left\{\left[\begin{array}{lll}
A_{1} & \bar{A}_{1} & B_{1}
\end{array}\right],\left[\begin{array}{lll}
A_{2} & \bar{A}_{2} & B_{2}
\end{array}\right], \ldots,\left[\begin{array}{lll}
A_{q} & \bar{A}_{q} & B_{q}
\end{array}\right]\right\},
\end{aligned}
$$

in which Co indicates the convex hull and $\left[\begin{array}{lll}A_{i} & \bar{A}_{i} & B_{i}\end{array}\right]$ are vertices of the convex hull. The nonnegative coefficients $\lambda_{i}(k)(i=1,2, \ldots, q)$ for each time $k$ satisfies the following:

$$
\begin{array}{r}
A(k) \bar{A}(k) B(k)]=\sum_{i=1}^{q} \lambda_{i}(k)\left[\begin{array}{lll}
A_{i} & \bar{A}_{i} & B_{i}
\end{array}\right], \\
\sum_{i=1}^{q} \lambda_{i}(k)=1 .
\end{array}
$$

In this paper we aims at designing the following controller for system in (1):

$$
u(k)=K(k) x(k),
$$

with the performance index as follows at every time $k$ :

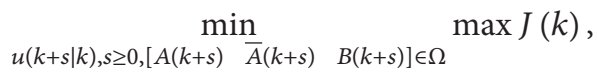

where

$$
\begin{array}{r}
J(k) \triangleq \sum_{s=0}^{\infty}\left\{\|x(k+s \mid k)\|_{\mathrm{Q}}^{2}+\|u(k+s \mid k)\|_{R}^{2}\right\}, \\
x(k+s+1 \mid k)=A(k+s) x(k+s \mid k) \\
+\bar{A}(k+s) x(k+s-d(k) \mid k) \\
+B(k+s) u(k+s \mid k), \\
-\bar{u} \leq u(k+s \mid k)=K(k) x(k+s \mid k) \leq \bar{u}, \\
s \in[0, \infty),
\end{array}
$$

where $Q$ and $R$ are known positive definite symmetric weighting matrices, $x(k+s \mid k)$ denotes the predicted state at time $k+s$ and $u(k+s \mid k)$ denotes the control signal at time $k+s$, when $x(k-s \mid k)=x(k-s)(s \geq 0)$. Based on the concept of MPC, before the next sampling time comes, we just implement the first compute input signal $u(k \mid k)$. Then we repeat the aforementioned optimization problem after updating it with the actual state.

Remark 1. The input-output technique is one of the most effective ways to handle the time delay, which was presented in the robust control theory $[13,14]$. Before using the approach to dispose time-varying delay, a proper approximation with small error for $x(k-d(k))$ should be found. In $[15,16]$, the authors used different variants of the state variable as the approximation of $x(k-d(k))$. In this paper, we utilize $\left(x\left(k-d_{m}\right)+x\left(k-d_{M}\right)\right) / 2$ as the approximation of $x(k-d(k))$.

Next, the lower and upper bounds $d_{m}$ and $d_{M}$ are used to estimate $x(k-d(k))$. The two-term approximation $(x(k-$ $\left.\left.d_{m}\right)+x\left(k-d_{M}\right)\right) / 2$ leads to the following error:

$$
\begin{aligned}
\sigma_{d_{0}}(k) & =\frac{2}{d_{0}}\left\{x(k-d(k))-\frac{1}{2}\left[x\left(k-d_{m}\right)+x\left(k-d_{M}\right)\right]\right\} \\
& =\frac{1}{d_{0}}\left[\sum_{i=k-d_{M}}^{k-d_{m}-1} \beta(i) \varsigma_{d_{0}}(i)\right],
\end{aligned}
$$


where $d_{0}=d_{M}-d_{m}, \varsigma_{d_{0}}(i) \triangleq x(i+1)-x(i)$, and

$$
\beta(i) \triangleq \begin{cases}1, & i \leq k-d(k)-1 \\ -1, & i>k-d(k)-1 .\end{cases}
$$

Then, system (1) is replaced by the following one:

$$
\begin{aligned}
x(k+1)= & A(k) x(k) \\
& +\frac{1}{2} \bar{A}(k)\left[x\left(k-d_{m}\right)+x\left(k-d_{M}\right)+d \sigma_{d_{0}}(k)\right] \\
& +B(k) u(k) \\
= & A(k) x(k)+\frac{1}{2} \bar{A}(k) x\left(k-d_{m}\right) \\
& +\frac{1}{2} x\left(k-d_{M}\right)+\frac{d_{0}}{2} d \sigma_{d_{0}}(k)+B(k) u(k) .
\end{aligned}
$$

Now let $x(k+s+1 \mid k)=x(k+s \mid k)+y(k+s \mid k)$. Then we gain the following:

$$
\begin{aligned}
& x\left(k+s-d_{m} \mid k\right)=x(k+s \mid k)-\sum_{i=1}^{d_{m}} y(k+s-i \mid k), \\
& x\left(k+s-d_{M} \mid k\right)=x(k+s \mid k)-\sum_{i=1}^{d_{M}} y(k+s-i \mid k) .
\end{aligned}
$$

As in [17], (8) can be converted into the following equivalent descriptor form:

$$
\begin{aligned}
& {[A(k+s)+B(k+s) K(k)+\bar{A}(k+s)-I] x(k+s \mid k)} \\
& \quad-\frac{1}{2} \bar{A}(k+s) \sum_{i=1}^{d_{m}} y(k+s-i \mid k) \\
& \quad-\frac{1}{2} \bar{A}(k+s) \sum_{i=1}^{d_{M}} y(k+s-i \mid k) \\
& +\frac{d_{0}}{2} \sigma_{d_{0}}(k) \bar{A}(k+s)-y(k+s \mid k)=0 .
\end{aligned}
$$

We introduce the following lemma for our main result.

Lemma 2. According to [18], suppose that $\alpha \in R^{n_{a}}, \beta \in R^{n_{b}}$, and $N \in R^{n_{a} \times n_{b}}$; then for any matrices $X \in R^{n_{a} \times n_{a}}, Y \in R^{n_{a} \times n_{b}}$, and $Z \in R^{n_{b} \times n_{b}}$, the following inequality holds:

$$
-2 \alpha^{T} N \beta \leq\left[\begin{array}{l}
\alpha \\
\beta
\end{array}\right]^{T}\left[\begin{array}{cc}
X & Y-N \\
* & Z
\end{array}\right]\left[\begin{array}{l}
\alpha \\
\beta
\end{array}\right],
$$

where $\left[\begin{array}{ll}X & Y \\ * & Z\end{array}\right] \geq 0$

\section{Main Results}

The following function is introduced in order to obtain the main results

$$
\begin{aligned}
V(x & (k+s \mid k)) \\
= & x^{T}(k+s \mid k) P_{1} x(k+s \mid k) \\
& +\sum_{\theta=1}^{d_{m}} \sum_{r=1}^{\theta} y^{T}(k+s-r \mid k) G_{1} y(k+s-r \mid k) \\
& +\sum_{\theta=1}^{d_{M}} \sum_{r=1}^{\theta} y^{T}(k+s-r \mid k) G_{2} y(k+s-r \mid k) \\
& +\sum_{r=1}^{d_{m}} x^{T}(k+s-r \mid k) H_{1} x(k+s-r \mid k) \\
& +\sum_{r=1}^{d_{M}} x^{T}(k+s-r \mid k) H_{2} x(k+s-r \mid k) .
\end{aligned}
$$

For every $[A(k+s) \quad \bar{A}(k+s) \quad B(k+s)] \in \Omega, s \geq 0$ and satsifies the following:

$$
\begin{aligned}
& V(x(k+s+1 \mid k))-V(x(k+s \mid k)) \\
& \quad \leq-\left[\|x(k+s \mid k)\|_{Q}^{2}+\|u(k+s \mid k)\|_{R}^{2}\right] ;
\end{aligned}
$$

we can obtain the upper bound of $J(k)$. Since $J(k)$ should be limited, one can get $x(\infty \mid k)=0$. Then, one can get $y(\infty \mid$ $k)=0$. Therefore, $V(x(\infty \mid k))=0$. It follows that $-V(x(k \mid$ $k)) \leq-J(k)$, by calculating the sum of inequality (17) from $s=0$ to $s=\infty$. Hence

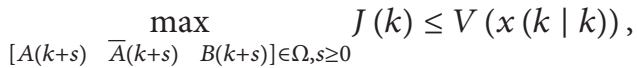

where

$$
\begin{aligned}
V(x(k \mid k))= & x^{T}(k \mid k) P_{1} x(k \mid k) \\
& +\sum_{\theta=1}^{d_{m}} \sum_{r=1}^{\theta} y^{T}(k-r \mid k) G_{1} y(k-r \mid k) \\
& +\sum_{\theta=1}^{d_{M}} \sum_{r=1}^{\theta} y^{T}(k-r \mid k) G_{2} y(k-r \mid k) \\
& +\sum_{i=1}^{d_{m}} x^{T}(k-r \mid k) H_{1} x(k-r \mid k) \\
& +\sum_{i=1}^{d_{M}} x^{T}(k-r \mid k) H_{2} x(k-r \mid k) .
\end{aligned}
$$

Therefore, it can be seen from (18) that we transform the organical min-max optimization problem in (6)-(9) into the following optimization problem which can minimize the upper bound of $V(x(k \mid k))$ as follows:

$$
\min _{K(k), P_{1}, G_{1}, G_{2}, H_{1}, H_{2}} V(x(k \mid k)) .
$$

Equations (9), (14), and (17) are the constraint conditions. 
Theorem 3. Considering the uncertain system in (1) with timevarying delay and input constrains (2), if there exist matrices $X>0, Y, Z, \bar{K}, U_{1}>0, U_{2}>0, U_{3}>0, U_{4}>0, \bar{W}_{1}, \bar{W}_{2}, \bar{W}_{3}$, and $E$ with appropriate dimensions and the scalar $\gamma>0$, the following problem is solvable:

$$
\min _{\gamma, X, Y, Z, \bar{K}, U_{1}, U_{2}, U_{3}, U_{4}, \bar{W}_{1}, \bar{W}_{2}, \bar{W}_{3}, E} \gamma,
$$

subject to

$$
\begin{aligned}
& {\left[\begin{array}{ll}
\Gamma_{1} & \Gamma_{2} \\
* & \Gamma_{3}
\end{array}\right] \geq 0} \\
& {\left[\begin{array}{cccccccccc}
\Theta_{1} & \Theta_{2} & 0 & 0 & Z^{T} & d_{m} Z^{T} & d_{M} Z^{T} & X & X^{T} Q^{1 / 2} & \bar{K}^{T} R^{1 / 2} \\
* & \Theta_{3} & \Theta_{4} & \Theta_{5} & Y^{T} & d_{m} Y^{T} & d_{M} Y^{T} & 0 & 0 & 0 \\
* & * & -U_{3} & 0 & 0 & 0 & 0 & 0 & 0 & 0 \\
* & * & * & -U_{4} & 0 & 0 & 0 & 0 & 0 & 0 \\
* & * & * & * & -X & 0 & 0 & 0 & 0 & 0 \\
* & * & * & * & * & -d_{m} U_{1} & 0 & 0 & 0 & 0 \\
* & * & * & * & * & * & -d_{M} U_{2} & 0 & 0 & 0 \\
* & * & * & * & * & * & * & -U_{3}-U_{4} & 0 & 0 \\
* & * & * & * & * & * & * & * & -\gamma I & 0 \\
* & * & * & * & * & * & * & * & * & -\gamma I
\end{array}\right] \leq 0,} \\
& {\left[\begin{array}{ccc}
\bar{W}_{1} & \bar{W}_{2} & 0 \\
* & \bar{W}_{3} & \varepsilon \bar{A}_{\sigma} U_{1} \\
* & * & U_{1}
\end{array}\right] \geq 0,} \\
& {\left[\begin{array}{ccc}
\bar{W}_{1} & \bar{W}_{2} & 0 \\
* & \bar{W}_{3} & \varepsilon \bar{A}_{\sigma} U_{2} \\
* & * & U_{2}
\end{array}\right] \geq 0,} \\
& {\left[\begin{array}{ll}
E & \bar{K} \\
* & X
\end{array}\right] \geq 0, \quad E_{i i} \leq \bar{u}_{i}^{2}, \quad i=1, \ldots, n_{u} \text {, }}
\end{aligned}
$$

where

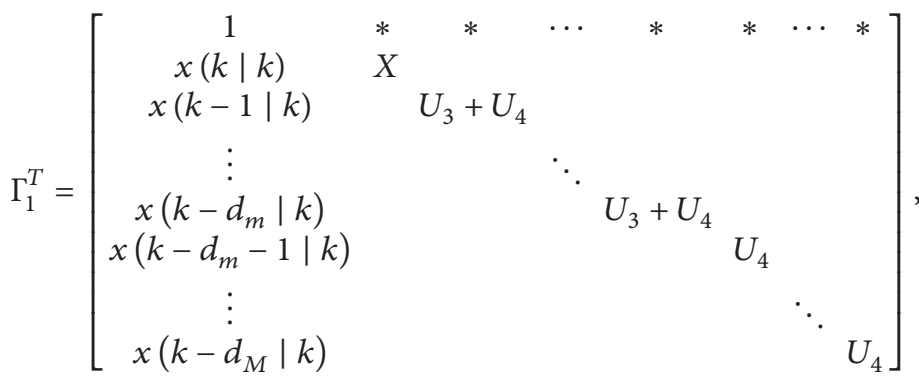

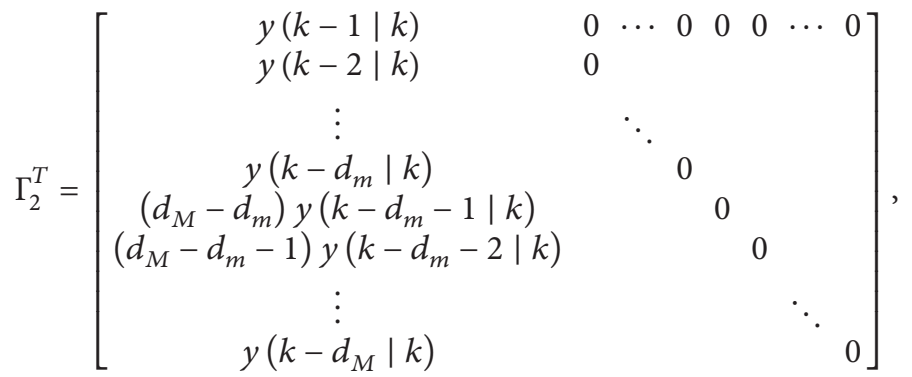




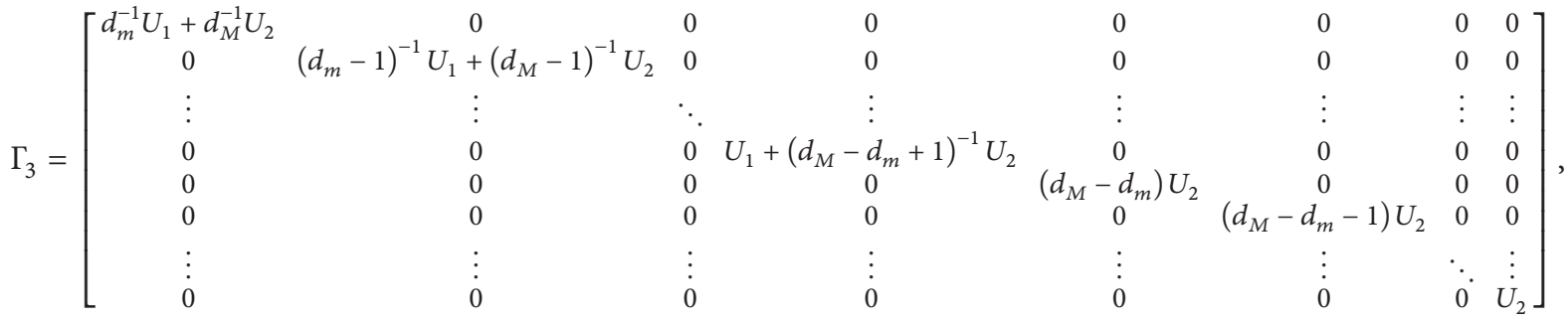

$$
\begin{aligned}
& \begin{array}{c}
\Theta_{1}=Z+Z^{T}+d_{m} \bar{W}_{1}+d_{M} \bar{W}_{1}, \\
\Theta_{2}=X\left(A_{\sigma}^{T}+2 \varepsilon A_{\sigma}^{T}-I\right)+Y+\bar{K}^{T} B_{\sigma}^{T}-Z^{T}+d_{m} \bar{W}_{2}+d_{M} \bar{W}_{2},
\end{array} \\
& \Theta_{3}=-Y-Y^{T}+d_{m} \bar{W}_{3}+d_{M} \bar{W}_{3}, \\
& \Theta_{4}=(1-\varepsilon) \bar{A}_{\sigma} U_{3}, \quad \Theta_{5}=-\varepsilon \bar{A}_{\sigma} U_{4}, \\
& \sigma=1, \ldots, q,
\end{aligned}
$$

and $\bar{u}_{i}$ indicates the ith element of $\bar{u}$ and $E_{i i}$ represents the ith diagonal element of $E$; then the upper bound $V(x(k \mid k))$ of the desired performance index is minimized by the MPC law $u(k+$ $s \mid k)=\bar{K} X^{-1} x(k+s \mid k), s \geq 0$.

Proof. Set $X=\gamma P_{1}^{-1}, U_{1}=\gamma S_{1}^{-1}, U_{2}=\gamma S_{2}^{-1}, U_{3}=\gamma H_{1}^{-1}$ and $U_{4}=\gamma H_{2}^{-1}$. Then, the problem of minimizing $V(k \mid k)$ can be regarded as follows:

$$
\min _{\gamma, P_{1}, G_{1}, G_{2}, H_{1}, H_{2}} \gamma
$$

subject to

$$
\begin{aligned}
V(x(k \mid k))= & x^{T}(k \mid k) P_{1} x(k \mid k) \\
& +\sum_{\theta=1}^{d_{m}} \sum_{r=1}^{\theta} y^{T}(k-r \mid k) G_{1} y(k-r \mid k) \\
& +\sum_{\theta=1}^{d_{M}} \sum_{r=1}^{\theta} y^{T}(k-r \mid k) G_{2} y(k-r \mid k) \\
& +\sum_{i=1}^{d_{m}} x^{T}(k-r \mid k) H_{1} x(k-r \mid k) \\
& +\sum_{i=1}^{d_{M}} x^{T}(k-r \mid k) H_{2} x(k-r \mid k) \leq \gamma .
\end{aligned}
$$

We can easily deduce (21) and (22) by using the Schur complement. According to the definition of $V(x(k+s \mid k))$ in (16), one can obtain the following:

$$
\begin{aligned}
& V(x(k+s+1 \mid k))-V(x(k+s \mid k)) \\
&= 2 x^{T}(k+s \mid k) P_{1} y(k+s \mid k) \\
&+x^{T}(k+s \mid k)\left(H_{1}+H_{2}\right) x(k+s \mid k)
\end{aligned}
$$

$$
\begin{aligned}
& +y^{T}(k+s \mid k)\left(P_{1}+d_{m} G_{1}+d_{M} G_{2}\right) y(k+s \mid k) \\
& -\sum_{r=1}^{d_{m}} y^{T}(k+s-r \mid k) G_{1} y(k+s-r \mid k) \\
& -\sum_{r=1}^{d_{M}} y^{T}(k+s-r \mid k) G_{2} y(k+s-r \mid k) \\
& -x^{T}\left(k+s-d_{m} \mid k\right) H_{1} x\left(k+s-d_{m} \mid k\right) \\
& -x^{T}\left(k+s-d_{M} \mid k\right) H_{1} x\left(k+s-d_{M} \mid k\right) .
\end{aligned}
$$

By Lemma 2 and the descriptor system (14), we have

$$
\begin{aligned}
& 2 x^{T}(k+s \mid k) P_{1} y(k+s \mid k) \\
& =2 \eta^{T}(k+s \mid k) P^{T} \\
& \times\left\{\left[\begin{array}{c}
y(k+s \mid k) \\
\Theta_{6}
\end{array}\right]-\sum_{i=1}^{d_{m}}\left[\begin{array}{c}
0 \\
\frac{1}{2} \bar{A}(k+s)
\end{array}\right] y(k+s-i \mid k)\right. \\
& \left.-\sum_{i=1}^{d_{M}}\left[\begin{array}{c}
0 \\
\frac{1}{2} \bar{A}(k+s)
\end{array}\right] y(k+s-i \mid k)\right\} \\
& \leq 2 \eta^{T}(k+s \mid k) P^{T} \\
& \times\left[\begin{array}{cc}
0 & I \\
A(k+s)+B(k+s) K(k)-I & -I
\end{array}\right] \eta(k+s \mid k)
\end{aligned}
$$

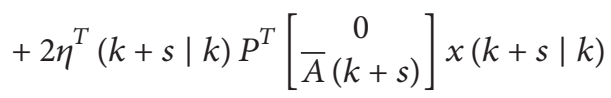

$$
\begin{aligned}
& +2 \eta^{T}(k+s \mid k) P^{T}\left[\begin{array}{c}
0 \\
\frac{d_{0}}{2} \sigma_{d_{0}} \bar{A}(k+s)
\end{array}\right]
\end{aligned}
$$




$$
\begin{aligned}
& +d_{m} \eta^{T}(k+s \mid k) W \eta(k+s \mid k) \\
& +d_{M} \eta^{T}(k+s \mid k) W \eta(k+s \mid k) \\
& +\sum_{i=1}^{d_{m}} y^{T}(k+s-r \mid k) G_{1} y(k+s-r \mid k) \\
& +\sum_{i=1}^{d_{M}} y^{T}(k+s-r \mid k) G_{2} y(k+s-r \mid k) \\
& +2 \eta^{T}(k+s \mid k)\left(M-P^{T}\left[\frac{1}{2} \bar{A}(k+s)\right]\right) \\
& \times\left(x(k+s \mid k)-x\left(k+s-d_{m} \mid k\right)\right) \\
& +2 \eta^{T}(k+s \mid k)\left(M-P^{T}\left[\frac{1}{2} \bar{A}(k+s)\right]\right) \\
& \times\left(x(k+s \mid k)-x\left(k+s-d_{M} \mid k\right)\right),
\end{aligned}
$$

where

$$
\begin{gathered}
\Theta_{6}=[A(k+s)+B(k+s) K(k)+\bar{A}(k+s)-I] \\
\times x(k+s \mid k)+\frac{d_{0}}{2} \sigma_{d_{0}} \bar{A}(k+s)-y(k+s \mid k), \\
\eta(k+s \mid k)=\left[\begin{array}{c}
x(k+s \mid k) \\
y(k+s \mid k)
\end{array}\right], \quad P=\left[\begin{array}{cc}
P_{1} & 0 \\
P_{2} & P_{3}
\end{array}\right],
\end{gathered}
$$

and matrices $W$ and $M$ satisfy the following conditions:

$$
\begin{aligned}
& {\left[\begin{array}{ll}
W & M \\
* & G_{1}
\end{array}\right] \geq 0,} \\
& {\left[\begin{array}{ll}
W & M \\
* & G_{2}
\end{array}\right] \geq 0 .}
\end{aligned}
$$

After substituting (31) into (30), we obtain the following inequality:

$$
\begin{aligned}
& V(x(k+s+1 \mid k))-V(x(k+s \mid k)) \\
& \leq \eta^{T}(k+s \mid k) \Phi \eta(k+s \mid k)+2 \eta^{T}(k+s \mid k) \\
& \quad \times\left(P^{T}\left[\frac{1}{2} \bar{A}(k+s)\right]-M\right) x\left(k+s-d_{m} \mid k\right) \\
& \quad+2 \eta^{T}(k+s \mid k)\left(P^{T}\left[\frac{1}{2} \bar{A}(k+s)\right]-M\right) \\
& \quad \times x\left(k+s-d_{M} \mid k\right) \\
& \quad-x^{T}\left(k+s-d_{m} \mid k\right) H_{1} x\left(k+s-d_{m} \mid k\right)
\end{aligned}
$$

$$
\begin{aligned}
& -x^{T}\left(k+s-d_{M} \mid k\right) H_{2} x\left(k+s-d_{M} \mid k\right) \\
& +2 \eta^{T}(k+s \mid k) P^{T}\left[\begin{array}{c}
0 \\
\frac{d_{0}}{2} \sigma_{d_{0}} \bar{A}(k+s)
\end{array}\right] \\
& =\varepsilon^{T}(k+s \mid k) \\
& \times\left[\begin{array}{ccc}
\Phi & P^{T}\left[\begin{array}{c}
0 \\
\frac{1}{2} \bar{A}(k+s)
\end{array}\right]-M & P^{T}\left[\begin{array}{c}
0 \\
\frac{1}{2} \bar{A}(k+s)
\end{array}\right]-M \\
* & -H_{1} & 0 \\
* & * & -H_{2}
\end{array}\right] \\
& \times \varepsilon(k+s \mid k) \\
& +2 \eta^{T}(k+s \mid k) P^{T}\left[\begin{array}{c}
0 \\
\frac{d_{0}}{2} \sigma_{d_{0}} \bar{A}(k+s)
\end{array}\right],
\end{aligned}
$$

where $\varepsilon^{T}(k+s \mid k)=\left[x^{T}(k+s \mid k) y^{T}(k+s \mid k) x^{T}(k+\right.$ $\left.\left.s-d_{m} \mid k\right) x^{T}\left(k+s-d_{M} \mid k\right)\right]$,

$$
\begin{aligned}
& \Phi=P^{T}\left[\begin{array}{cc}
0 & I \\
A(k+s)+B(k+s) K(k)-I & -I
\end{array}\right] \\
& +\left[\begin{array}{cc}
0 & I \\
A(k+s)+B(k+s) K(k)-I & -I
\end{array}\right]^{T} P \\
& +d_{m} W+d_{M} W+2\left[\begin{array}{ll}
M & 0
\end{array}\right]+2\left[\begin{array}{c}
M^{T} \\
0
\end{array}\right] \\
& +\left[\begin{array}{cc}
H_{1}+H_{2} & 0 \\
* & P_{1}+d_{m} G_{1}+d_{M} G_{2}
\end{array}\right], \\
& \sigma_{d_{0}}(k+s)=\frac{1}{d_{0}}\left[\sum_{i=k-d_{M}}^{k-d_{m}-1} \beta(i) \varsigma_{d_{0}}(i)\right] \\
& =\frac{1}{d_{0}}\left[\sum_{i=k+s-d_{M}}^{k-d_{0}-1+s} y(i) \beta(i)\right] \\
& \leq \frac{1}{d_{0}} \sum_{r=d_{m}+1}^{d_{M}} y(k+s-r) \\
& =\frac{1}{d_{0}}\left[\sum_{r=1}^{d_{M}} y(k+s-r)-\sum_{r=1}^{d_{m}} y(k+s-r)\right] . \\
& V(x(k+s+1 \mid k))-V(x(k+s \mid k)) \\
& \leq \varepsilon^{T}(k+s \mid k)
\end{aligned}
$$




$$
\begin{array}{ll}
\times\left[\begin{array}{ccc}
\Phi & P^{T}\left[\begin{array}{c}
0 \\
\frac{1}{2} \bar{A}(k+s)
\end{array}\right]-M & P^{T}\left[\begin{array}{c}
0 \\
\frac{1}{2} \bar{A}(k+s)
\end{array}\right]-M \\
* & -H_{1} & 0 \\
* & * & -H_{2}
\end{array}\right] & =\varepsilon^{T}(k+s \mid k) \\
\times \varepsilon(k+s \mid k) &
\end{array}
$$$$
+\eta^{T}(k+s \mid k) P^{T} \sum_{r=1}^{d_{M}}\left[\begin{array}{c}
0 \\
A \\
(k+s)
\end{array}\right] y(k+s-r \mid k)
$$$$
-\eta^{T}(k+s \mid k) P^{T} \sum_{r=1}^{d_{m}}\left[\begin{array}{c}
0 \\
A \\
(k+s)
\end{array}\right] y(k+s-r \mid k)
$$$$
=\varepsilon^{T}(k+s \mid k)
$$$$
\left.\times\left[\begin{array}{ccc}
\Phi & P^{T}\left[\begin{array}{c}
0 \\
\frac{1}{2} \bar{A}(k+s)
\end{array}\right]-M & P^{T}\left[\begin{array}{c}
0 \\
*
\end{array}\right. \\
* & -H_{1} & 0 \\
* & * & -H_{2}
\end{array}\right]-M\right]
$$

Replacing (17) with (37), we have

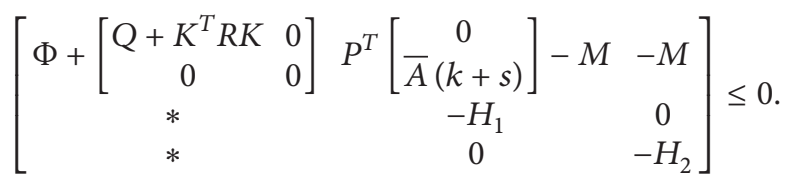

For obtaining LMI, we give the definition as follows:

$$
\begin{gathered}
M=\varepsilon P^{T}\left[\begin{array}{c}
0 \\
\bar{A}(k+s)
\end{array}\right], \quad \bar{K}=K X, \\
\gamma P^{-1}=\left[\begin{array}{cc}
X & 0 \\
Z & Y
\end{array}\right], \\
\bar{W}=\gamma\left(P^{-1}\right)^{T} W\left(P^{-1}\right)=\left[\begin{array}{cc}
\bar{W}_{1} & \bar{W}_{2} \\
* & \bar{W}_{3}
\end{array}\right] .
\end{gathered}
$$

$$
\begin{aligned}
& +\eta^{T}(k+s \mid k) P^{T}\left[\begin{array}{c}
0 \\
\bar{A}(k+s)
\end{array}\right] x\left(k+s-d_{m} \mid k\right) \\
& -\eta^{T}(k+s \mid k) P^{T}\left[\begin{array}{c}
0 \\
\bar{A}(k+s)
\end{array}\right] x\left(k+s-d_{M} \mid k\right)
\end{aligned}
$$

Pre- and postmultiplying (38), (33), and (34) by $\operatorname{diag}\left\{\gamma^{1 / 2}\left(P^{-1}\right)^{T}, \gamma^{-1 / 2} U_{3}, \gamma^{-1 / 2} U_{4}\right\}$ and $\operatorname{diag}\left\{\gamma^{1 / 2}\left(P^{-1}\right), \gamma^{-1 / 2}\right.$ $\left.U_{3}, \gamma^{-1 / 2} U_{4}\right\}, \operatorname{diag}\left\{\gamma^{1 / 2}\left(P^{-1}\right)^{T}, \gamma^{-1 / 2} U_{1}\right\}$ and $\operatorname{diag}\left\{\gamma^{1 / 2}\left(P^{-1}\right)\right.$, $\left.\gamma^{-1 / 2} U_{1}\right\}$, and $\operatorname{diag}\left\{\gamma^{1 / 2}\left(P^{-1}\right)^{T}, \gamma^{-1 / 2} U_{2}\right\}$ and $\operatorname{diag}\left\{\gamma^{1 / 2}\left(P^{-1}\right)\right.$, $\left.\gamma^{-1 / 2} U_{2}\right\}$, respectively, then (38) is equivalent to the following:

$$
\left[\begin{array}{cccccccccc}
\Theta_{7} & \Theta_{8} & 0 & 0 & Z^{T} & d_{m} Z^{T} & d_{M} Z^{T} & X & X^{T} Q^{1 / 2} & \bar{K}^{T} R^{1 / 2} \\
* & \Theta_{9} & \Theta_{10} & \Theta_{11} & Y^{T} & d_{m} Y^{T} & d_{M} Y^{T} & 0 & 0 & 0 \\
* & * & -U_{3} & 0 & 0 & 0 & 0 & 0 & 0 & 0 \\
* & * & * & -U_{4} & 0 & 0 & 0 & 0 & 0 & 0 \\
* & * & * & * & -X & 0 & 0 & 0 & 0 & 0 \\
* & * & * & * & * & -d_{m} U_{1} & 0 & 0 & 0 & 0 \\
* & * & * & * & * & * & -d_{M} U_{2} & 0 & 0 & 0 \\
* & * & * & * & * & * & * & -U_{3}-U_{4} & 0 & 0 \\
* & * & * & * & * & * & * & * & -\gamma I & 0 \\
* & * & * & * & * & * & * & * & * & -\gamma I
\end{array}\right] \leq 0
$$

where

$$
\begin{gathered}
\Theta_{7}=Z+Z^{T}+d_{m} \bar{W}_{1}+d_{M} \bar{W}_{1}, \\
\Theta_{8}=X\left(A^{T}(k+s)+2 \varepsilon A^{T}(k+s)-I\right) \\
+Y+\bar{K}^{T} B^{T}(k+s)-Z^{T}+d_{m} \bar{W}_{2}+d_{M} \bar{W}_{2}, \\
\Theta_{9}=-Y-Y^{T}+d_{m} \bar{W}_{3}+d_{M} \bar{W}_{3}, \\
\Theta_{10}=(1-\varepsilon) \bar{A}(k+s) U_{3}, \\
\Theta_{11}=-\varepsilon \bar{A}(k+s) U_{4} .
\end{gathered}
$$

Equation (33) is equivalent to the following:

$$
\left[\begin{array}{ccc}
\bar{W}_{1} & \bar{W}_{2} & 0 \\
* & \bar{W}_{3} & \varepsilon \bar{A} U_{1} \\
* & * & U_{1}
\end{array}\right] \geq 0,
$$

Equation (34) is equivalent to the following:

$$
\left[\begin{array}{ccc}
\bar{W}_{1} & \bar{W}_{2} & 0 \\
* & \bar{W}_{3} & \varepsilon \bar{A} U_{2} \\
* & * & U_{2}
\end{array}\right] \geq 0
$$

If and only if inequalities in (23)-(25) hold, respectively, inequalities in (40)-(43) are fulfilled for any 
$[A(k) \bar{A}(k) B(k)] \in \Omega$, for that $(40)-(43)$ are affine on the basis of matrices $[A(k) \bar{A}(k) B(k)]$.

Now we consider the input constraints (9) and discuss how to transform it into LMI. First we introduce the following invariant ellipsoid:

$$
\omega=\left\{z \in R^{n\left(2 d_{M}+1\right)} \mid z^{T} \Psi^{-1} z \leq 1\right\},
$$

where

$$
\begin{aligned}
& \Psi=\operatorname{diag}\left\{\begin{array}{llll}
X & U_{3}+U_{4} & \cdots & U_{3}+U_{4}
\end{array}\right. \\
& U_{4} \cdots U_{4} \quad d_{m}^{-1} U_{1}+d_{M}^{-1} U_{2} \\
& \left(d_{m}-1\right)^{-1} U_{1}+\left(d_{M}-1\right)^{-1} U_{2} \cdots \\
& U_{1}+\left(d_{M}-d_{m}+1\right)^{-1} U_{2}\left(d_{M}-d_{m}\right) U_{2} \\
& \left.\left(d_{M}-d_{m}-1\right) U_{2} \cdots U_{2}\right\} \\
& z=[x(k+s \mid k) x(k+s-1 \mid k) \cdots \\
& x\left(k+s-d_{m} \mid k\right) x\left(k+s-d_{m}-1 \mid k\right) \cdots \\
& x\left(k+s-d_{M} \mid k\right) y(k+s-1 \mid k) \\
& y(k+s-2 \mid k) \cdots y\left(k+s-d_{m} \mid k\right) \\
& y\left(k+s-d_{m}-1 \mid k\right) y\left(k+s-d_{m}-2 \mid k\right) \\
& \left.\ldots y\left(k+s-d_{M} \mid k\right)\right] \text {. }
\end{aligned}
$$

As discussed in [12], it is shown that

$$
\begin{aligned}
\max _{s \geq 0} & \left|u_{i}(k+s \mid k)\right|^{2} \\
& =\max _{s \geq 0}\left|\left(\bar{K} X^{-1} x(k+s \mid k)\right)_{i}\right|^{2} \\
& \leq \max \left|\left(\widehat{K} \Psi^{-1} z\right)_{i}\right|^{2} \\
& =\max \left|\left(\widehat{K} \Psi^{-1 / 2} \Psi^{-1 / 2} z\right)_{i}\right|^{2} \\
& \leq\left\|\left(\widehat{K} \Psi^{-1 / 2}\right)_{i}\right\|_{2}^{2}
\end{aligned}
$$

(using the Cauchy-Schwarz inequality)$$
=\left(\widehat{K} \Psi^{-1} \widehat{K}^{T}\right)_{i i},
$$

where $\widehat{K}=\left[\begin{array}{llll}\bar{K} & 0 & \cdots & 0\end{array}\right]$.

Therefore, there exists a symmetric matrix $E$ and the following inequality is satisfied:

$$
\left[\begin{array}{cc}
E & \widehat{K} \\
\widehat{K}^{T} & \Psi
\end{array}\right] \geq 0, \quad E_{i i} \leq \bar{u}_{i}^{2}, \quad i=1, \ldots, n_{u} .
$$

It is easily shown that (47) is equivalent to (26) with the definitions of $\widehat{K}$ and $\Psi$. This completes the proof.
Theorem 4. If the optimization problem in (21)-(26) is solved with a feasible solution, the MPC law designed in Theorem 3 can make the resulting closed-loop system robustly asymptotically stable.

Proof. At first, let one verify the feasibility of optimization problem (21)-(26). A feasible sequence $u(k+s \mid k), s \geq 0$ is supposed to exist in (21)-(26) at time $k$. At time $k+1$, we choose the following feasible control sequence obtained at time $k$ to guarantee the existence of feasible solutions:

$$
u(k+s+1 \mid k+1)=u^{*}(k+s+1 \mid k), \quad s \geq 0,
$$

where $u^{*}(k+s+1 \mid k)$ is a solution obtained at time $k$. The input constraint (9) at time $k+1$ is satisfied. It indicates the feasibilities of the optimization problem at all future instants. Then the stability of the closed-loop system is given. Let $P_{1}^{*}(k), G_{1}^{*}(k), G_{2}^{*}(k), H_{1}^{*}(k), H_{2}^{*}(k)$, and $P_{1}^{*}(k+1), G_{1}^{*}(k+1)$, $G_{2}^{*}(k+1), H_{1}^{*}(k+1)$, and $H_{2}^{*}(k+1)$ indicate the optimal values at time $k$ and $k+1$, respectively. Now we take the following quadratic function into account:

$$
\begin{aligned}
V^{*}(x(k \mid k))= & x^{T}(k \mid k) P_{1}^{*}(k) x(k \mid k) \\
& +\sum_{\theta=1}^{d_{m}} \sum_{r=1}^{\theta} y^{T}(k-r \mid k) G_{1}^{*}(k) y(k-r \mid k) \\
& +\sum_{\theta=1}^{d_{M}} \sum_{r=1}^{\theta} y^{T}(k-r \mid k) G_{2}^{*}(k) y(k-r \mid k) \\
& +\sum_{r=1}^{d_{m}} x^{T}(k-r \mid k) H_{1}^{*}(k) x(k-r \mid k) \\
& +\sum_{r=1}^{d_{M}} x^{T}(k-r \mid k) H_{2}^{*}(k) x(k-r \mid k) .
\end{aligned}
$$

Since $P_{1}^{*}(k+1), G_{1}^{*}(k+1), G_{2}^{*}(k+1), H_{1}^{*}(k+1)$, and $H_{2}^{*}(k+1)$ are optimal, while $P_{1}^{*}(k), G_{1}^{*}(k), G_{2}^{*}(k), H_{1}^{*}(k)$, and $H_{2}^{*}(k)$ are also feasible at time $k+1$, we have

$$
\begin{aligned}
& V^{*}(x(k+1 \mid k+1)) \\
& =x^{T}(k+1 \mid k+1) P_{1}^{*}(k+1) x(k+1 \mid k+1) \\
& +\sum_{\theta=1}^{d_{m}} \sum_{r=1}^{\theta} y^{T}(k+1-r \mid k) G_{1}^{*}(k+1) \\
& \quad \times y(k+1-r \mid k+1) \\
& +\sum_{\theta}^{d_{M}} \sum_{r=1}^{\theta} y^{T}(k+1-r \mid k) G_{2}^{*}(k+1) \\
& \quad \times y(k+1-r \mid k+1)
\end{aligned}
$$




$$
\begin{aligned}
& +\sum_{r=1}^{d_{m}} x^{T}(k+1-r \mid k+1) H_{1}^{*}(k+1) \\
& \times x(k+1-r \mid k+1) \\
& +\sum_{r=1}^{d_{M}} x^{T}(k+1-r \mid k+1) H_{2}^{*}(k+1) \\
& \times x(k+1-r \mid k+1) \\
& \leq x^{T}(k+1 \mid k+1) P_{1}^{*}(k) x(k+1 \mid k+1) \\
& +\sum_{\theta=1}^{d_{m}} \sum_{r=1}^{\theta} y^{T}(k+1-r \mid k) G_{1}^{*}(k) \\
& \times y(k+1-r \mid k+1) \\
& +\sum_{\theta=1}^{d_{M}} \sum_{r=1}^{\theta} y^{T}(k+1-r \mid k) G_{2}^{*}(k) \\
& \times y(k+1-r \mid k+1) \\
& +\sum_{r=1}^{d_{m}} x^{T}(k+1-r \mid k+1) H_{1}^{*}(k) \\
& \times x(k+1-r \mid k+1) \\
& +\sum_{r=1}^{d_{M}} x^{T}(k+1-r \mid k+1) H_{2}^{*}(k) \\
& \times x(k+1-r \mid k+1) .
\end{aligned}
$$

Furthermore, from (17), it follows that $V(x(k+1 k))-V(x(k \mid$ $k)) \leq 0$. Thus, one can obtain

$$
\begin{aligned}
& x^{T}(k+1 \mid k) P_{1}^{*}(k) x(k+1 \mid k) \\
& +\sum_{\theta=1}^{d_{m}} \sum_{r=1}^{\theta} y^{T}(k+1-r \mid k) G_{1}^{*}(k) \\
& \quad \times y(k+1-r \mid k) \\
& +\sum_{\theta=1}^{d_{M}} \sum_{r=1}^{\theta} y^{T}(k+1-r \mid k) G_{2}^{*}(k) \\
& \quad \times y(k+1-r \mid k) \\
& +\sum_{r=1}^{d_{m}} x^{T}(k+1-r \mid k) H_{1}^{*}(k) \\
& \times x(k+1-r \mid k) \\
& +\sum_{r=1}^{d_{M}} x^{T}(k+1-r \mid k) H_{2}^{*}(k) \\
& \times x(k+1-r \mid k)
\end{aligned}
$$

$$
\begin{aligned}
\leq & x^{T}(k \mid k) P_{1}^{*}(k) x(k \mid k) \\
& +\sum_{\theta=1}^{d_{m}} \sum_{r=1}^{\theta} y^{T}(k-r \mid k) G_{1}^{*}(k) y(k-r \mid k) \\
& +\sum_{\theta=1}^{d_{M}} \sum_{r=1}^{\theta} y^{T}(k-r \mid k) G_{2}^{*}(k) y(k-r \mid k) \\
& +\sum_{r=1}^{d_{m}} x^{T}(k-r \mid k) H_{1}^{*}(k) x(k-r \mid k) \\
& +\sum_{r=1}^{d_{M}} x^{T}(k-r \mid k) H_{2}^{*}(k) x(k-r \mid k) \\
= & V^{*}(x(k \mid k)) .
\end{aligned}
$$

From the definition of $y(k+s \mid k)$ above, it follows that $y(k+$ $1-s \mid k+1)=x(k+2-s \mid k+1)-x(k+1-s \mid$ $k+1)=x(k+2-s \mid k)-x(k+1-s \mid k), s=1, \ldots, d_{0}$, as $x(k+1 \mid k+1)=x(k+1 \mid k)$ and $x(k+1-s \mid k+1)=$ $x(k+1-s \mid k), s=1, \ldots, d_{0}$, for $[A(k) \bar{A}(k) B(k)] \in \Omega$ at each time $k \cdot x(k+1-s \mid k+1), s=1, \ldots, d_{0}$, and $y(k+1-s \mid$ $k+1), s=1, \ldots, d_{0}$ in (50) are replaced with $x(k+1-s \mid k)$, $s=1, \ldots, d_{0}$, and $y(k+1-s \mid k), s=1, \ldots, d_{0}$, respectively. Thus, according to (51), one can get

$$
V^{*}(x(k+1 \mid k+1)) \leq V^{*}(x(k \mid k)) .
$$

It is shown that the Lyapunov function $V^{*}(x(k \mid k))$ is bounded and monotonically nonincreasing. Therefore, $x(k)$ approaches 0 when $k$ approaches $\infty$. And the proof is completed.

Remark 5. According to the condition in Theorem 3, $x(k-r \mid$ $k)=x(k-r)$ and $y(k-r \mid k)=x(k-r+1)-x(k-r)$, $r=1, \ldots, d_{0}$, are decided by the previous time and remained fixed. Thus, for given $\varepsilon$, (21)-(26) can be regarded as a LMI optimization problem. And we can obtain the numerical solution efficiently in terms of LMIs.

Remark 6. Different from the delay-independent MPC methods in $[4,12]$, Theorem 3 indicates that the proposed MPC technique is decided by the value of delay. The delay considered in this paper is unknown, while the delay is known in the reference [10].

\section{Simulation Results}

In this section, the truck-trailer system used in [4] is provided to illustrate the effectiveness of the method proposed in this paper:

$$
\begin{gathered}
\dot{x}_{1}(t)=-\delta \frac{v \bar{t}}{L t_{0}} x_{1}(t)-(1-\delta) \frac{v \bar{t}}{L t_{0}} x_{1}(t-\tau)+\frac{v \bar{t}}{l t_{0}} u(t), \\
\dot{x}_{2}(t)=\delta \frac{v \bar{t}}{L t_{0}} x_{1}(t)+(1-\delta) \frac{v \bar{t}}{L t_{0}} x_{1}(t-\tau),
\end{gathered}
$$




$$
\begin{aligned}
\dot{x}_{3}(t)=\frac{v \bar{t}}{t_{0}} \sin [ & x_{2}(t)+\delta \frac{v \bar{t}}{2 L} x_{1}(t) \\
& \left.+(1-\delta) \frac{v \bar{t}}{2 L} x_{1}(t-\tau)\right],
\end{aligned}
$$

where the variables $x_{1}(t), x_{2}(t), x_{3}(t)$, and $u(t)$, respectively, denote the angle difference between the trailer and truck, the angel of the trailer, the $y$-coordinate of the rear end of the trailer, and the steering angel. Delays exist in $x_{1} ;|u(t)| \leq$ $\pi$. The system parameters are given as $r=2.8 \mathrm{~m}, L=5.5 \mathrm{~m}$, $v=-1.0 \mathrm{~m} / \mathrm{s}, \bar{t}=2.0 \mathrm{~s}$, and $t_{0}=0.5 \mathrm{~s}$. The constant $\delta$ denotes the retarded coefficient varying $\delta \in[0,1]$. In our numerical example, we would like to suppose $\delta=0.7$. The time-delay system with nonlinearity is transformed into discrete-time polytopic uncertain system as follows with the sampling time $T=0.1 \mathrm{~s}:$

$$
x(k+1)=A(k) x(k)+\bar{A}(k) x(k-d(k))+B(k) u(k),
$$

where $[A(k) \bar{A}(k) B(k)]$ satisfies (3) with $q=2$, and

$$
\begin{gathered}
A_{1}=\left[\begin{array}{ccc}
1.0509 & 0 & 0 \\
-0.0509 & 1.0000 & 0 \\
0.0509 & -0.4000 & 1.0000
\end{array}\right], \\
\bar{A}_{1}=\left[\begin{array}{ccc}
0.0218 & 0 & 0 \\
-0.0218 & 0 & 0 \\
0.0218 & 0 & 0
\end{array}\right], \quad B_{1}=\left[\begin{array}{c}
-0.1429 \\
0 \\
0
\end{array}\right], \\
A_{2}=\left[\begin{array}{ccc}
1.0509 & 0 & 0 \\
-0.0509 & 1.0000 & 0 \\
0.0810 & -0.6366 & 1.0000
\end{array}\right], \\
\bar{A}_{2}=\left[\begin{array}{ccc}
0.0218 & 0 & 0 \\
-0.0218 & 0 & 0 \\
0.0347 & 0 & 0
\end{array}\right], \quad B_{2}=\left[\begin{array}{c}
-0.1429 \\
0 \\
0
\end{array}\right] .
\end{gathered}
$$

We choose the initial state $x(0)=\left[\begin{array}{lll}0.5 & 0.75 \pi & -5\end{array}\right]^{T}$, the delay lower bound $d_{m}=1$, the delay upper bound $d_{m}=$ 10 , the input and state weighting matrices $R=1$, and $Q=\operatorname{diag}\{10,10,10\}$, respectively. In addition, we choose $\varepsilon=1.5$. Figure 1 shows the state response of the open-loop system which is unstable. In order to stabilize the system, we implement the MPC strategy at each step to design the controller $u(k+s \mid k)=K x(k+s \mid k), s \geq 0$. It can be found from Theorem 3 that the MPC state-feedback law is obtained as follows:

$$
K=\left[\begin{array}{lll}
2.3627 & -1.0863 & 0.1195
\end{array}\right] .
$$

In order to show the advantages of this paper over the existing results $[4,19]$, we give the comparison simulation results as follows. Figures 2(a) and 2(b) plot the state response of the closed loop system acquired through the MPC methods presented in this paper and in [4], respectively. It is obvious that the system employing our MPC method performs better with the faster response, smaller overshoot, stronger

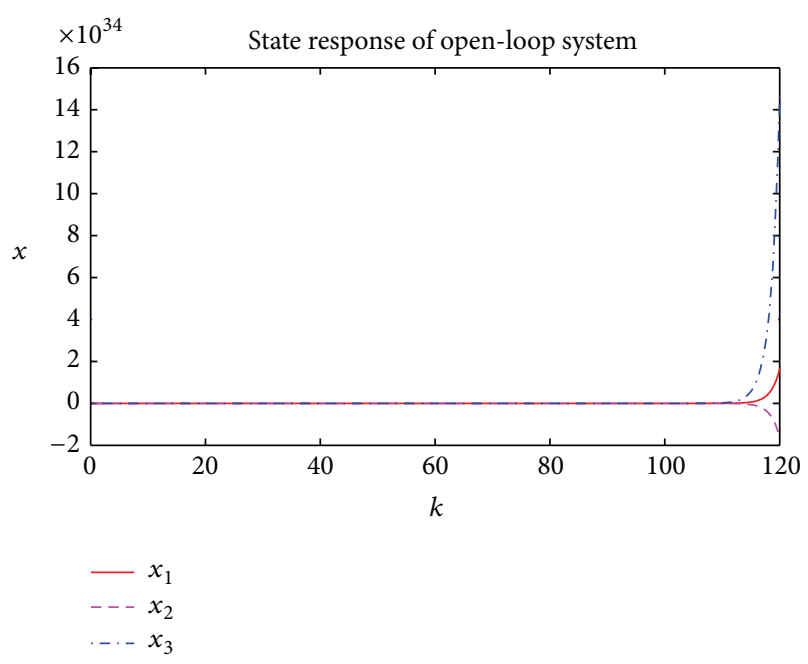

FIGURE 1: State response of open-loop system.

robustness, and so on than the existence results in [4]. Figures 3(a) and 3(b) show the control inputs obtained by the MPC algorithms in this paper and in [4], respectively. It is shown that both of them are not out of the input constraint. However, in this paper, the value of the input is smaller and the input trajectory is smoother. Figure 4 show the cost function trajectories gained by the two MPC methods. In addition, it is found that the method presented in [19] is not feasible in general numerical example.

\section{Conclusion}

The problem of MPC for a class of uncertain systems subject to time-varying delays and input constraints has been studied in this paper. $x(k-d(k))$ is estimated by its lower and upper bounds. Then the system is transformed into an equivalent descriptor system. Using some advanced method, the MPC law has been designed to guarantee the resulting closed-loop system to be asymptotically stable. The merits of the MPC approach proposed in this work have been demonstrated by a practical example. In future work, the proposed MPC technique will be applied to some kinds of systems, such as Markovian jumping systems [20] and fuzzy systems [21-24].

\section{Conflict of Interests}

The authors declare that there is no conflict of interests regarding the publication of this paper.

\section{Acknowledgment}

This work was partially supported by the National Natural Science Foundation of China (61304003 and 61304002). 

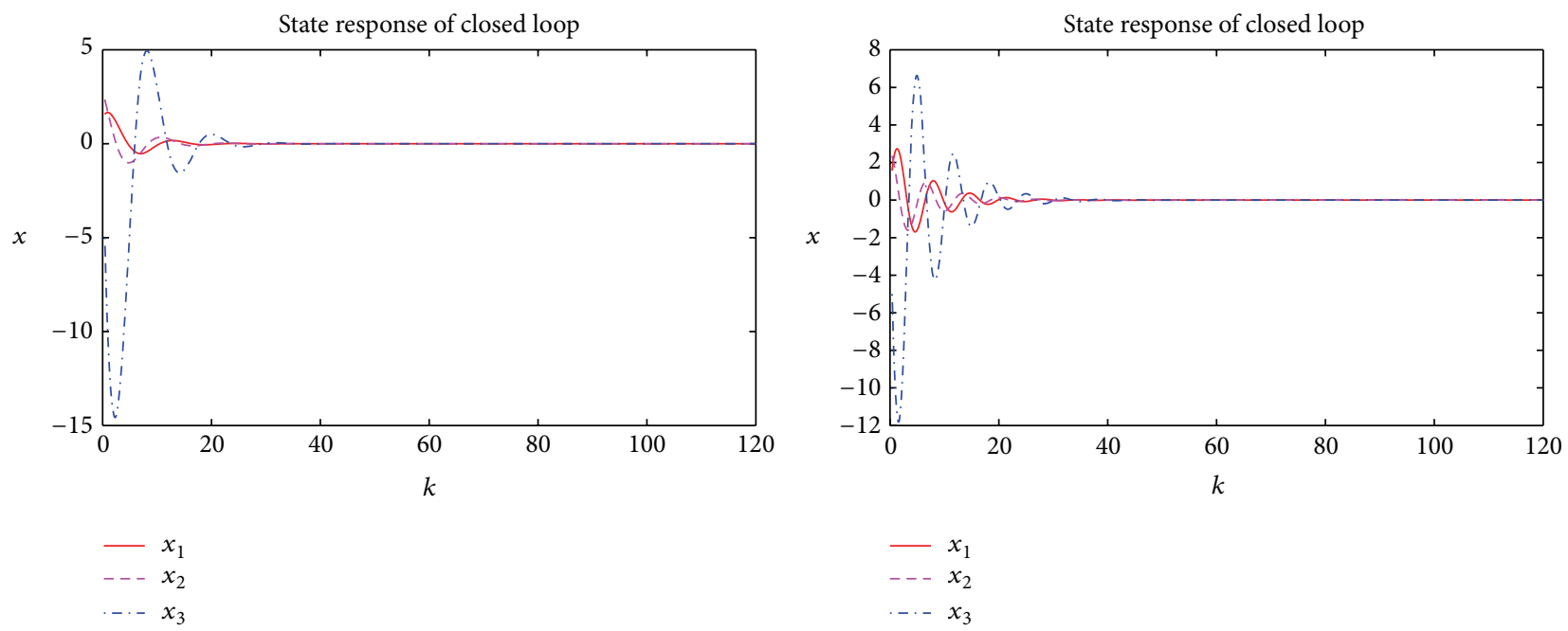

(a)

(b)

FIGURE 2: Comparison of the closed-loop state responses ((a) using our method and (b) using the method proposed in [4]).

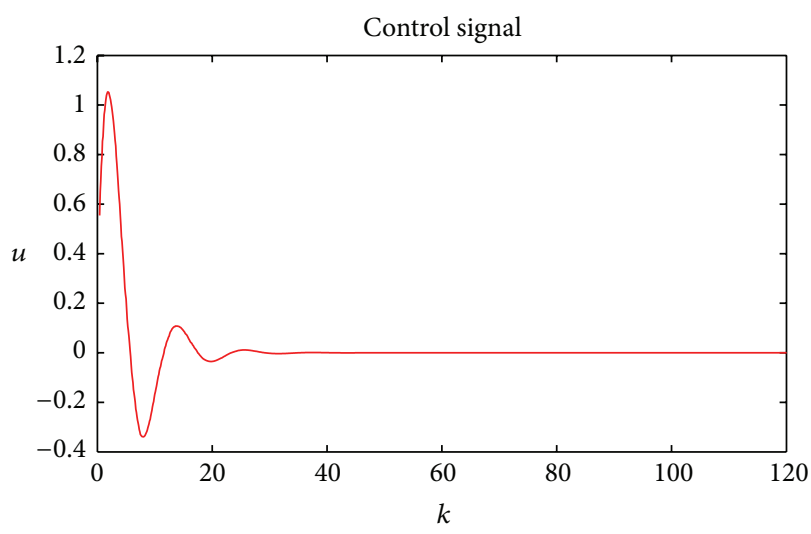

(a)

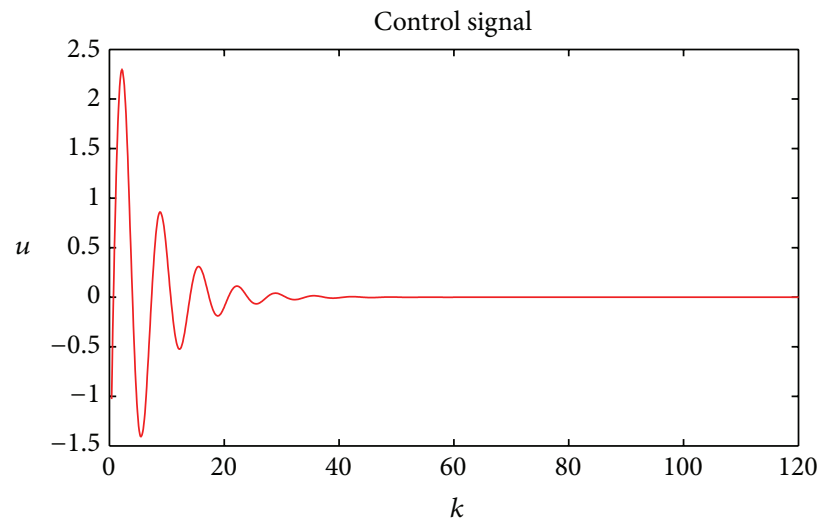

(b)

Figure 3: Comparison of the cost function ((a) using our method and (b) using the method proposed in [4]).

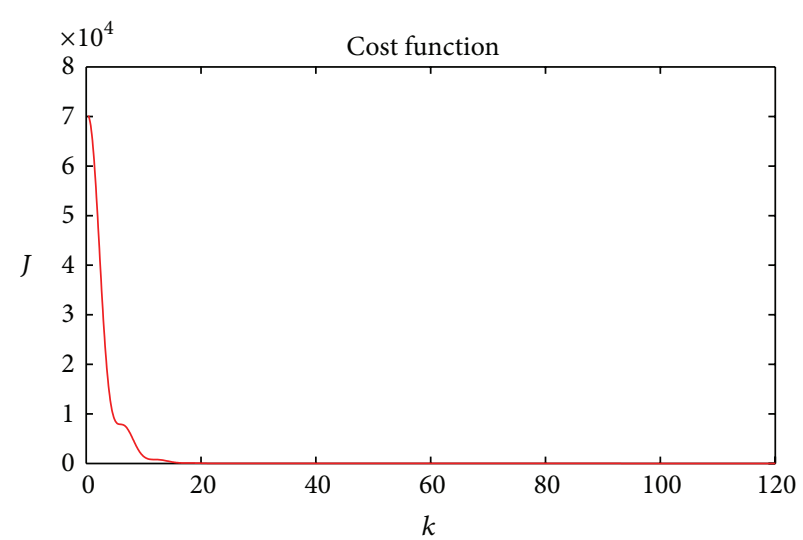

(a)

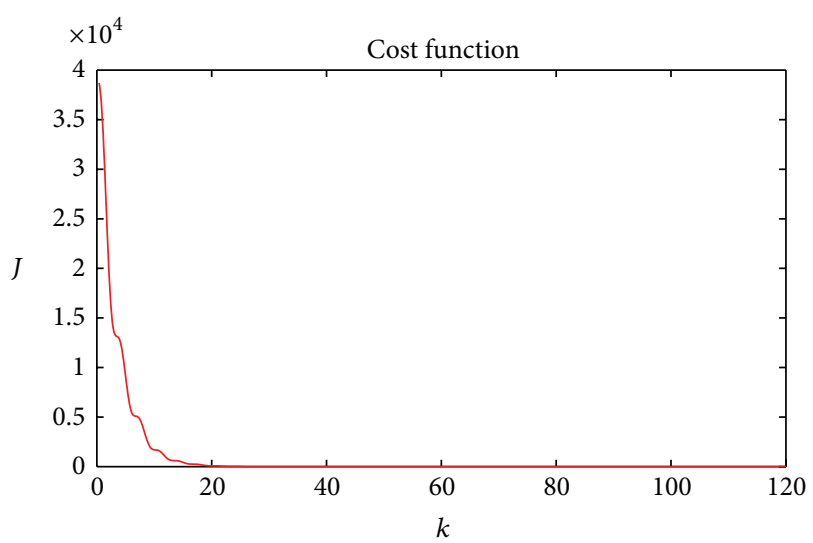

(b)

FIGURE 4: Comparison of the cost function ((a) using our method and (b) using the method proposed in [4]). 


\section{References}

[1] J. Richalet, A. Rault, J. L. Testud, and J. Papon, "Model predictive heuristic control. Applications to industrial processes," Automatica, vol. 14, no. 5, pp. 413-428, 1978.

[2] M. Morari and J. H. Lee, "Model predictive control: past, present and future," Computers and Chemical Engineering, vol. 23, no. 45, pp. 667-682, 1999.

[3] P. D. Christofides, R. Scattolini, D. Muñoz de la Peña, and J. Liu, "Distributed model predictive control: a tutorial review and future research directions," Computers and Chemical Engineering, vol. 51, pp. 21-41, 2013.

[4] S. C. Jeong and P. Park, "Constrained MPC algorithm for uncertain time-varying systems with state-delay," IEEE Transactions on Automatic Control, vol. 50, no. 2, pp. 257-263, 2005.

[5] M. Liu, D. W. C. Ho, and Y. Niu, "Stabilization of Markovian jump linear system over networks with random communication delay," Automatica, vol. 45, no. 2, pp. 416-421, 2009.

[6] C. Lin, Z. Wang, and F. Yang, "Observer-based networked control for continuous-time systems with random sensor delays," Automatica, vol. 45, no. 2, pp. 578-584, 2009.

[7] L. Wu and W. X. Zheng, "Weighted $H_{\infty}$ model reduction for linear switched systems with time-varying delay," Automatica, vol. 45, no. 1, pp. 186-193, 2009.

[8] Z.-G. Wu, P. Shi, H. Su, and J. Chu, "Dissipativity analysis for discrete-time stochastic neural networks with time-varying delays," IEEE Transactions on Neural Networks and Learning Systems, vol. 24, no. 3, pp. 345-355, 2013.

[9] H. Li, X. Jing, and H. R. Karimi, "Output-feedback-based $H_{\infty}$ control for vehicle suspension systems with control delay," IEEE Transactions on Industrial Electronics, vol. 61, no. 1, pp. 436-446, 2014.

[10] Y.-J. Shi, T.-Y. Chai, H. Wang, and C.-Y. Su, "Delay-dependent robust model predictive control for time-delay systems with input constraints," in Proceedings of the American Control Conference (ACC '09), pp. 4880-4885, June 2009.

[11] C. Wen, J. Zhou, Z. Liu, and H. Su, "Robust adaptive control of uncertain nonlinear systems in the presence of input saturation and external disturbance," IEEE Transactions on Automatic Control, vol. 56, no. 7, pp. 1672-1678, 2011.

[12] M. V. Kothare, V. Balakrishnan, and M. Morari, "Robust constrained model predictive control using linear matrix inequalities," Automatica, vol. 32, no. 10, pp. 1361-1379, 1996.

[13] J. Doyle, "Robust constrained model predictive control using linear matrix inequalities," IEE Proceedings D: Control Theory and Applications, vol. 129, no. 6, pp. 242-250, 1982.

[14] K. Zhou, J. C. Doyle, K. Glover et al., Robust and Optimal Control, vol. 40, Prentice Hall, Upper Saddle River, NJ, USA, 1996.

[15] K. Gu, Y. Zhang, and S. Xu, "Small gain problem in coupled differential-difference equations, time-varying delays, and direct Lyapunov method," International Journal of Robust and Nonlinear Control, vol. 21, no. 4, pp. 429-451, 2011.

[16] X. Li and H. Gao, "A new model transformation of discrete-time systems with time-varying delay and its application to stability analysis," IEEE Transactions on Automatic Control, vol. 56, no. 9, pp. 2172-2178, 2011.

[17] W.-H. Chen, Z.-H. Guan, and X. Lu, "Delay-dependent guaranteed cost control for uncertain discrete-time systems with delay," IEE Proceedings-Control Theory and Applications, vol. 150, no. 4, pp. 412-416, 2003.
[18] Y. S. Moon, P. Park, W. H. Kwon, and Y. S. Lee, "Delaydependent robust stabilization of uncertain state-delayed systems," International Journal of Control, vol. 74, no. 14, pp. 14471455, 2001.

[19] L. Jianxiong, F. Yiming, and S. Shengli, "Robust MPC algorithm for discrete-time systems with time-varying delay and nonlinear perturbations," in Proceedings of the IEEE 29th Chinese Control Conference (CCC '10), pp. 3128-3133, IEEE, Beijing, China, July 2010.

[20] H. Li, H. Gao, and P. Shi, "New passivity analysis for neural networks with discrete and distributed delays," IEEE Transactions on Neural Networks, vol. 21, no. 11, pp. 1842-1847, 2010.

[21] H. Li, X. Jing, H.-K. Lam, and P. Shi, "Fuzzy sampled-data control for uncertain vehicle suspension systems," IEEE Transactions on Cybernetics, vol. 44, no. 7, pp. 1111-1126, 2014.

[22] H. Li, J. Yu, C. Hilton, and H. Liu, "Adaptive sliding-mode control for nonlinear active suspension vehicle systems using TS fuzzy approach," IEEE Transactions on Industrial Electronics, vol. 60, no. 8, pp. 3328-3338, 2013.

[23] S. Tong, C. Liu, and Y. Li, "Fuzzy-adaptive decentralized output-feedback control for large-scale nonlinear systems with dynamical uncertainties," IEEE Transactions on Fuzzy Systems, vol. 18, no. 5, pp. 845-861, 2010.

[24] S. Tong, Y. Li, and Y. Liu, "Observer-based adaptive fuzzy backstepping control for a class of stochastic nonlinear strictfeedback systems," IEEE Transactions on Systems, Man, and Cybernetics B: Cybernetics, vol. 41, no. 6, pp. 1693-1704, 2011. 


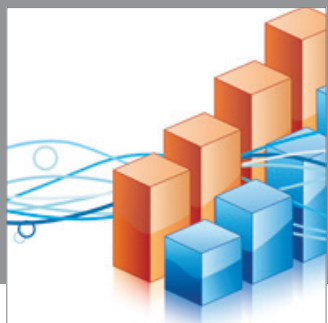

Advances in

Operations Research

mansans

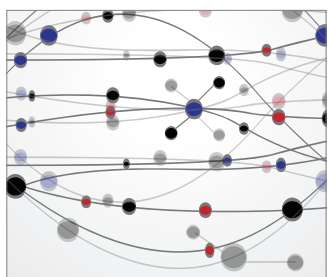

The Scientific World Journal
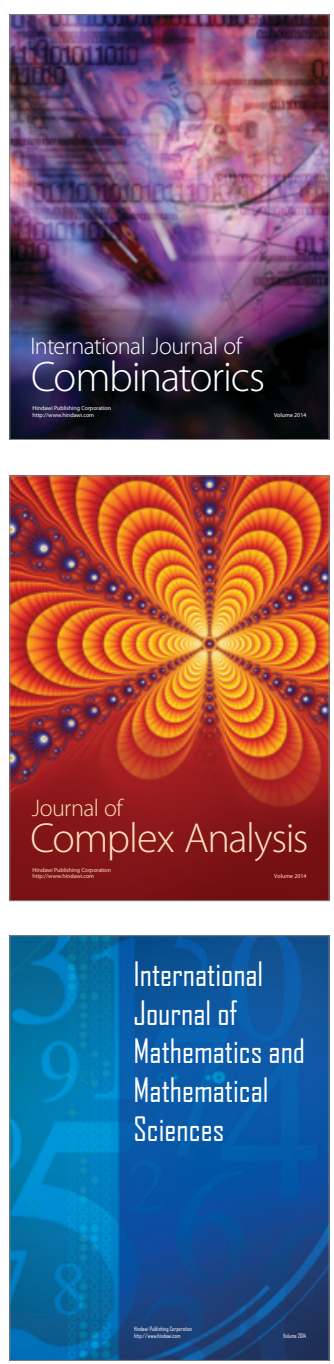
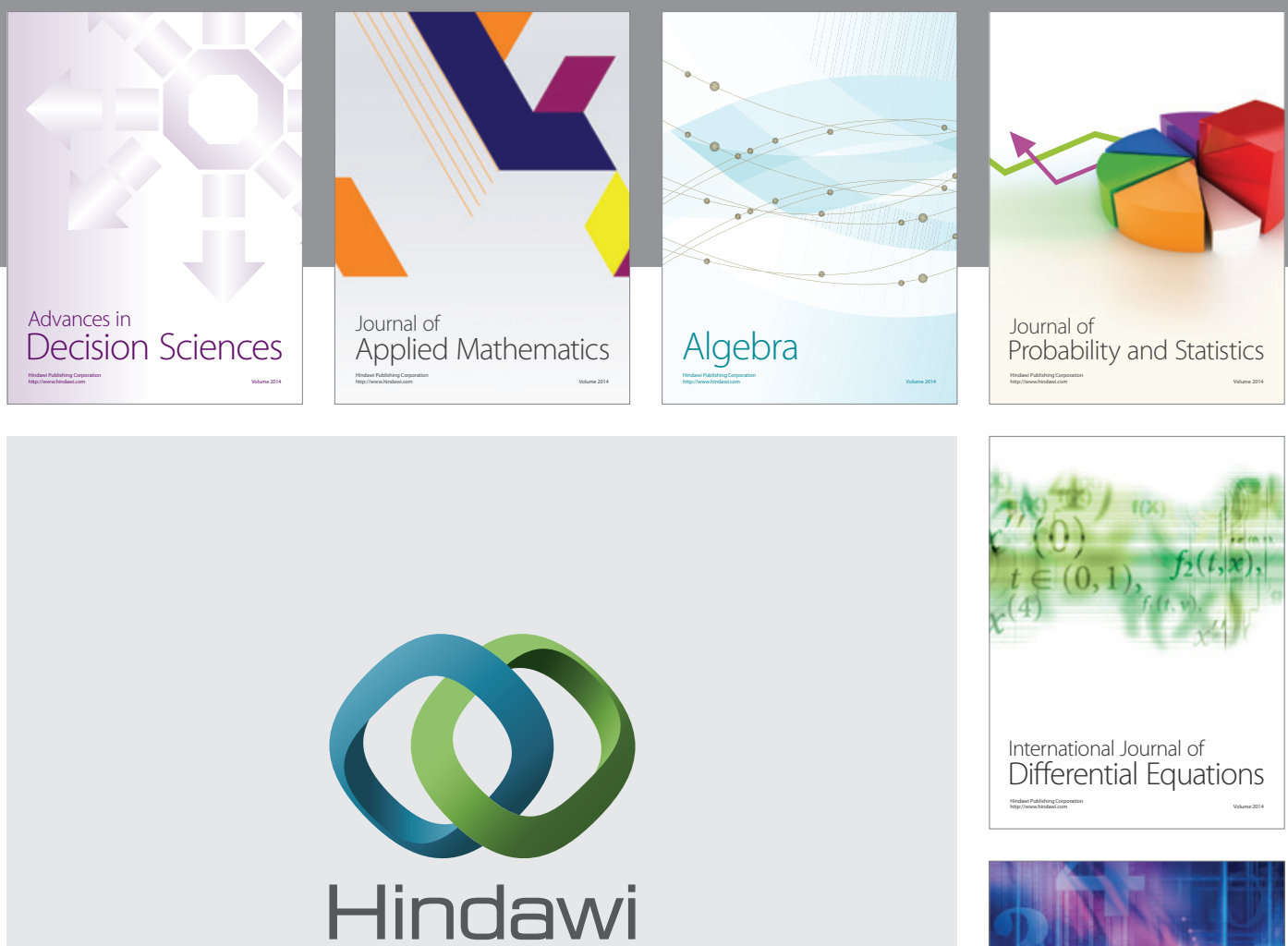

Submit your manuscripts at http://www.hindawi.com
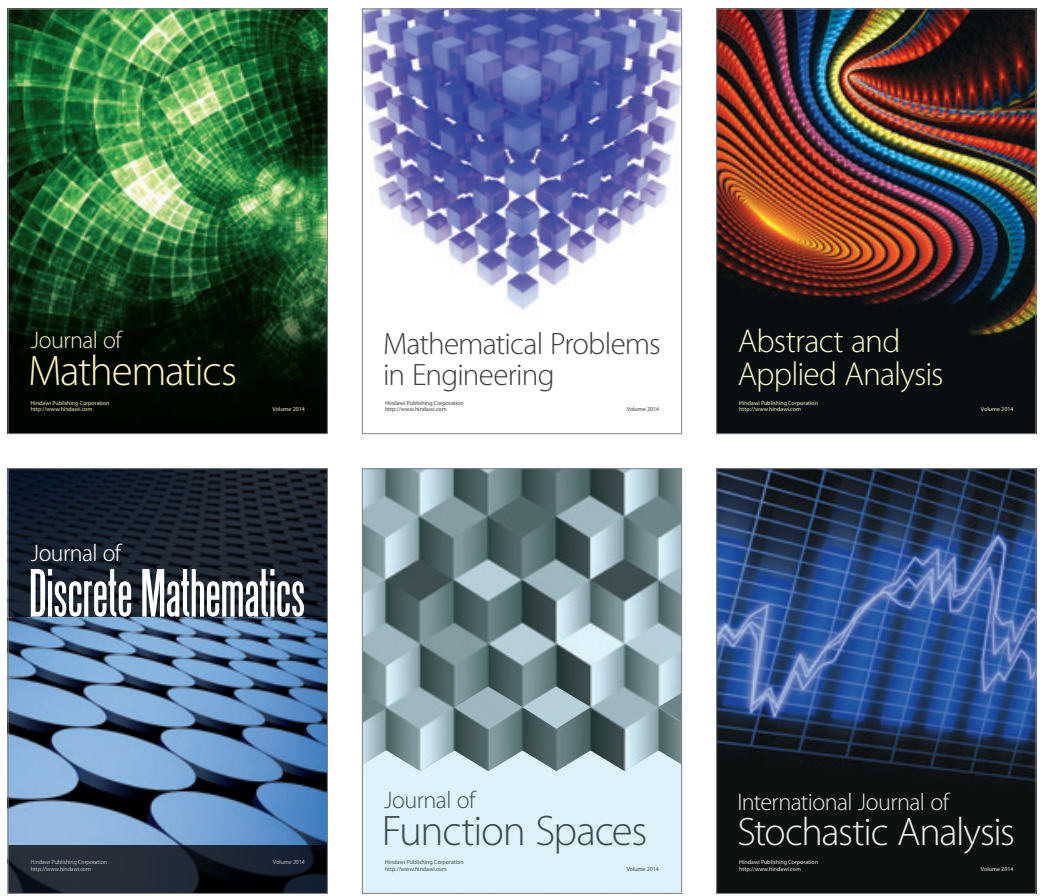

Journal of

Function Spaces

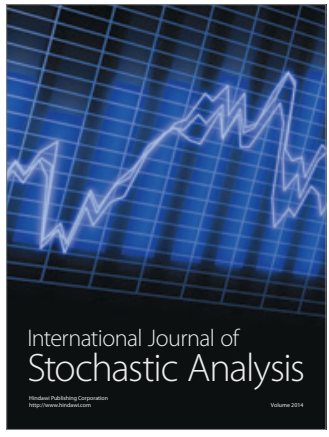

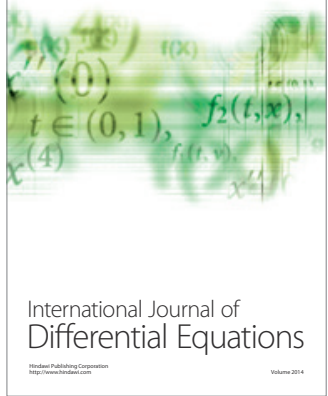
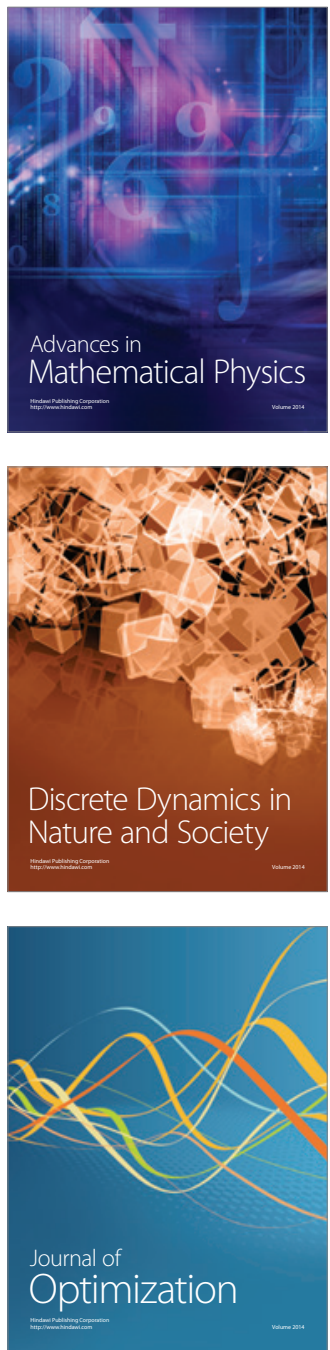